\title{
Diagnostics of the 3D Shape of a Rock Failure Zone by Electrical Measurements on the Earth's Surface
}

\author{
Dmitry Sirota \\ T.F. Gorbachev Kuzbass State Technical University \\ KuzSTU \\ Kemerovo, Russia \\ dmsirota@yandex.ru
}

\begin{abstract}
In a number of previously published papers by D. Sirota and V. Ivanov, it is theoretically and experimentally shown that the electrical measurements on the earth's surface can determine the approximate size and depth of the hearth of a man-made earthquake and a rock bursts in coal seams at depths of the hearth to several hundred meters. To predict the energy of the upcoming seismic event and determine the location of the event, it is necessary to determine the size, shape and orientation of the hearth.
\end{abstract}

Keywords - exploration geophysics, diagnostic problem, interpretation of geophysical data, rock burst, integral equation of the first kind, inverse ill-posed problem, tikhonov regularization method

\section{INTRODUCTION}

Theoretical, laboratory and mine experimental studies conducted at Kuzbass state technical University have produced the following main results $[1-4]$.

The process of preparing a rock bursts or man-made earthquake is kinetic, i.e., a time-course process consisting of the stages of scattered accumulation of microcracks and the stage of localization of destruction, when a macro-rupture is formed, and at the first stage the process is a Markov random process, and at the last it is subject to Hurst statistics, i.e. it is not Markov. At the first stage, the process is adequately described on the basis of the kinetic theory of strength of S. N. Zhurkov [5]. In the process of birth or slip each crack separation or shear in rocks of different mineral composition carries at its apex charge of one sign and dipole moment, which quickly relax after stopping the crack (for $10^{-4}-10^{-11}$ sec.). For different rocks charges range from $10^{-11}$ to $10^{-13}$ KL per unit length of crack front, with the main mechanisms of charge and dipole moment are the piezoelectric effect of rocks, diffusion of charged point defects of the structure (vacancies and interstitial ions) in the field of mechanical stresses of a moving crack, adhesion on the contact of mineral grains composing the rock. Each arising and spreading crack corresponds to the pulse of electromagnetic emission, and the ensemble of arising cracks creates a background of electromagnetic radiation, well recorded in mine conditions long before the mining impact. Each arising and spreading crack corresponds to the pulse of electromagnetic emission. The ensemble of arising cracks creates a background of electromagnetic radiation, well recorded in mine conditions long before localization of the rock bursts zone. It can be by electrical measurements on the earth surface or in the atmosphere during avalanche-unstable cracking, when in each cubic centimeter of the hearth according to the concentration criterion of destruction SN.

\author{
Vadim Ivanov \\ T.F. Gorbachev Kuzbass State Technical University \\ KuzSTU \\ Kemerovo, Russia \\ v.ivanov@nc-vostnii.ru
}

Zhurkov accumulates up to $10^{13}$ microcracks with sizes from $10^{-4}$ to $10^{-3} \mathrm{~m}$.

In this article let us investigate the inverse problem of determination the 3D shape of the rock burst or man-made earthquakes. The solution of this problem will be based on the using regularization technique by A. N. Tikhonov $[6,76]$ and transform to solving the Fredholm-Urisohn integral equation of the first kind [ $8-15]$. Let us apply the random search method "simulated annealing" (SA) [16, 17] in conjunction with the conjugate gradient method (CG) [18 20] for solving the multiextremal auxiliary optimization problem.

\section{FORMULATION AND SOLUTION OF THE DIRECT PROBLEM}

Let us assume that the electric field is generated by a 3Dsource of arbitrary shape $V_{p}$ and the containing space is the homogeneous isotropic two-layers with plane-parallel borders and the specific resistance $\rho_{1}, \rho_{2}$ and the field source is in the first layer. Let us first calculate the field of a point source (Fig. 1) in the coordinate system Oxyz, which is located in point source.

As well as known, the potential on the second layer and on the earth's surface from the point source can be calculated by the formula [4]:

$$
u_{2}=\frac{C}{\left[x_{M}^{2}+y_{M}^{2}+z_{M}^{2}\right]^{1 / 2}},
$$

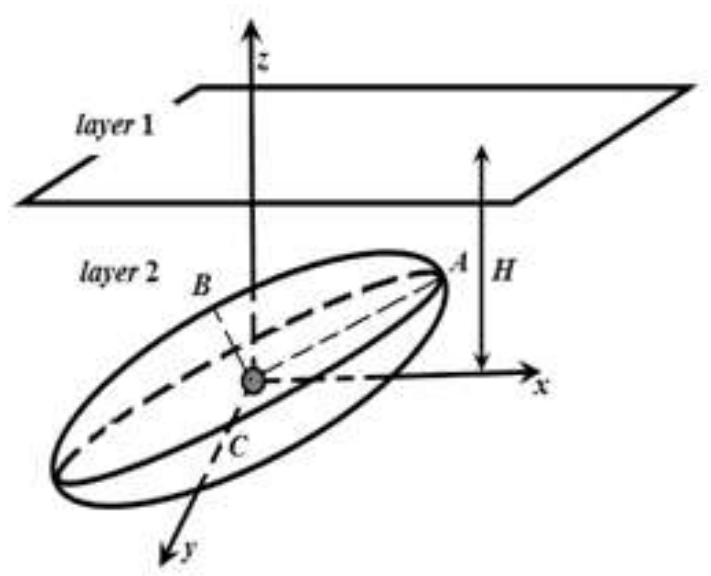

Fig. 1. To calculation the field in the atmosphere from the failure zone 
where $C-$ is the coefficient characterizing the source; $x_{M}, y_{M}$ $z_{M}-$ are the point of measurement coordinates on the earth's surface.

In [ $1-3]$ we calculated the potential for a flat shape and a cylinder. In this paper we will consider the general case of a 3D body and ellipsoid for testing program for solving inverse problem.

If we want to calculate the potential from the $3 \mathrm{D}$ source we must to integrate the (1) as

$$
U_{2}=C \int_{V_{P}} \frac{d V_{P}}{M P}
$$

where $V_{P}$ is the 3D body (ellipsoid Fig. 1); $x_{P}, y_{P}, z_{P}$ are the coordinates of the point $P$ inside the focal zone, which in (2) is the external integration; $x_{M}, y_{M}, z_{M}$ are the coordinates of measurement point on the earth's surface, $M P=\left[\left(x_{P}-x_{M}\right)^{2}+\left(y_{P}-y_{M}\right)^{2}+\left(z_{P}-z_{M}\right)^{2}\right]^{1 / 2}$ is the distance between $M$ and $P$.

Let us transform the (2) to a dimensionless form by notations $w / z_{M}=\bar{w}$, where the dimensions of a quantity of $w$ is the meter as in $[2,3]$. After that the elementary volume $d V_{P}$ is converted to the following dimensionless quantity $d V_{p}=z_{M}^{3} d \bar{V}_{p}$ and the (2) will be transformed to

$$
U_{2}=C \cdot z_{M}^{2} \cdot \int_{V_{P}} \frac{d \bar{V}_{P}}{\left[r^{2}+(\bar{z} P-1)^{2}\right]^{1 / 2}}
$$

where $r^{2}=\left(\bar{x}_{P}-\bar{x}_{M}\right)^{2}+\left(\bar{y}_{P}-\bar{y}_{M}\right)^{2}$.

Below for simplicity let us not to write line over the dimensionless quantities.

To calculate the three dimensional integral, we define the boundary of the region as a function $R(\varphi, \Psi)$ in the spherical coordinate system as follows: $x_{p}=\rho \sin \psi \cos \varphi$ $y_{p}=\rho \sin \psi \sin \varphi, z_{p}=\rho \cos \psi, 0 \leq \varphi \leq 2 \pi, 0 \leq \psi \leq \pi$ and $d V_{p}=\rho^{2} \sin \psi d \varphi d \psi d \rho$.

Thus we can rewrite the $3 \mathrm{D}$ integral (3) as

$$
\iiint_{V_{P}} \frac{d V_{p}}{M P}=\int_{0}^{2 \pi} d \varphi \int_{0}^{\pi} \sin \psi d \psi \int_{0}^{R(\varphi, \psi)} \frac{\rho^{2} d \rho}{\sqrt{\rho^{2}+b \rho+a}}
$$

where $b=-2\left[x_{M} \sin \psi \cos \varphi+y_{M} \sin \psi \sin \varphi+z_{M} \cos \psi\right]$, $a=x_{M}^{2}+y_{M}^{2}+z_{M}^{2}$.

The inner integral $I$ over variable $\rho$ we can calculate as

$$
\begin{aligned}
& I=\left(\frac{R(\varphi, \psi)}{2}-\frac{3 b}{4}\right) \times \sqrt{R^{2}(\varphi, \psi)+b R(\varphi, \psi)+a}+ \\
& +\left(\frac{3 b^{2}}{8}-\frac{a}{2}\right)^{R(\varphi, \psi)} \int_{0}^{\sqrt{\rho^{2}+b \rho+a}}+\frac{3 b}{4} \sqrt{a}
\end{aligned}
$$

and the one more secondary integral $I_{l}$ is calculate as

$$
I_{1}=\left\{\begin{array}{c}
\ln \left|\frac{R R+R(\varphi, \psi)+0,5 b \mid, \quad \text { if } b^{2}-a \neq 0}{0,5 b+\sqrt{a}}\right| \frac{\sqrt{a}}{R(\varphi, \psi)-\sqrt{a} \mid, \quad \text { if } \quad b=2 \sqrt{a}} \\
\ln \left|\frac{R(\varphi, \psi)}{\sqrt{a}}-1\right|, \quad \text { if } \quad b=-2 \sqrt{a}
\end{array}\right.
$$

where $R R=\sqrt{R^{2}(\varphi, \psi)+b R(\varphi, \psi)+a}$.

To calculate the intensity of a field we can use the difference schemes of 2 nd order of accuracy:

$$
E_{x}=\frac{U(x+\Delta)-U(x-\Delta)}{2 \Delta}, E_{y}=\frac{U(y+\Delta)-U(y-\Delta)}{2 \Delta}
$$

Bellow we will test program for the modeling focal area as ellipsoid with the axes $A>B>C$. The dimensionless equation of it in spherical coordinate system will look like

$$
R(\varphi, \psi)=\frac{A B C}{h \sqrt{T_{1}^{2}+T_{2}^{2}+T_{3}^{2}}},
$$

where $\gamma$ is the angle from the interval $23^{0}<\gamma<65^{0}$, $T_{1}=(\sin \psi \cos \varphi \cos \gamma-\cos \psi \sin \gamma) B C, T_{2}=\sin \psi \sin \varphi A C$, $T_{3}=(\sin \psi \cos \varphi \sin \gamma+\cos \psi \cos \gamma) A B$

Let us calculate dimensionless potential and intensity for the ellipsoid as the field source with parameters $A=50$, $B=25, C=10, H=150 \mathrm{~m}, \gamma=30^{\circ}, 45^{\circ}, 60^{\circ}$ and measurement point with coordinates $y_{M}=0$, $x_{M} \in[-400 ; 400] \mathrm{m}$. The graphical results of calculations are in Fig 2, 3.

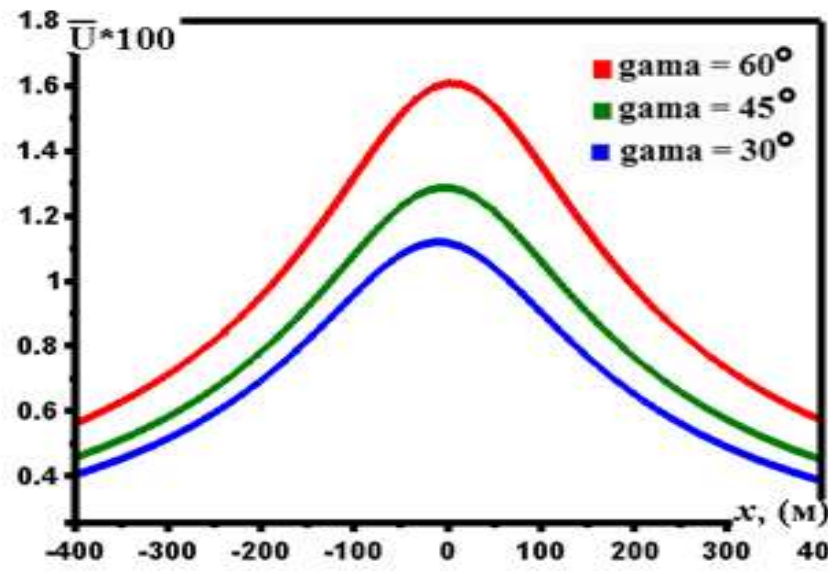

Fig. 2. The values of the dimensionalless potential of the field. 


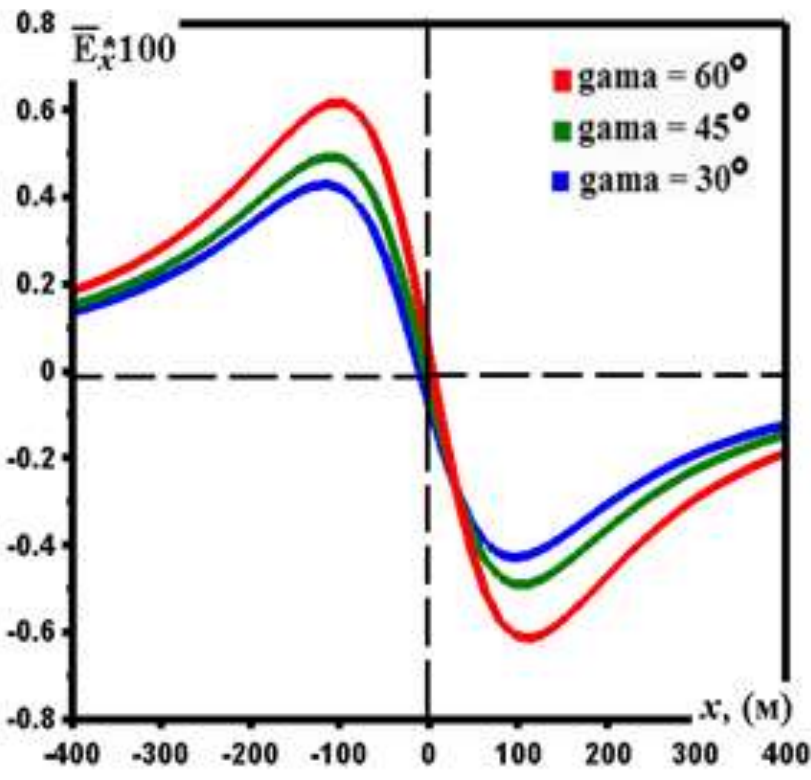

Fig. 3. The values of the dimensionalless field intensity

Thus, the problem of diagnostic the form of the 3D source based on electrical measurements on earth's surface has been reduce to the Fredholm-Urysohn integral equation of the first kind with respect to the unknown function $R(\varphi, \psi)$ :

$$
\int_{0}^{2 \pi} d \varphi \int_{0}^{\pi} \sin \psi \cdot F(M, R(\varphi, \psi)) d \psi=U^{*}
$$

where $F=\int_{0}^{R(\varphi, \psi)} \frac{\rho^{2} d \rho}{\sqrt{\rho^{2}+b \rho+a}}$ from the (5), $U^{*}-$ the experimental values of potential or intensity of field.

\section{SOLUTION OF THE INVERSE PROBLEM}

Let us introduce the solution of the inverse diagnostic problem for an unknown function $R(\varphi, \psi)$ based on finding the minimum of the A.N. Tikhonov's regularized functional $[6,7,8-15]$ :

$$
\Phi(R)=\mu\left(x_{M}, y_{M}, R\right)+\alpha \Omega(R) \rightarrow \min ,
$$

where $\mu=\sum_{x_{M}} \sum_{y_{M}}\left[A\left(x_{M}, y_{M}, R\right)-U^{*}\left(x_{M}, y_{M}\right)\right]^{2} \quad$ is the error in measuring the intensity on the earth's surface; $\Omega(R)=\int_{0}^{2 \pi} d \varphi \int_{0}^{\pi}\left[R^{2}(\varphi, \psi)+\left(\frac{\partial R(\varphi, \psi)}{\partial \varphi}\right)^{2}+\left(\frac{\partial R(\varphi, \psi)}{\partial \psi}\right)^{2}\right] d \psi$

is a stabilizing functional of the second order, $\alpha-$ is the regularization parameter $(5,6) ; A-$ is the direct modeling operator (3).

Let us solve the problem (8) with two type of search: exploratory and advance. As the exploratory we will use the method of random search "simulated annealing", as the advance we will use the conjugated gradient (CG) method.
Let us give an algorithm for the first method [16, 17].

Step 1. Create start value of $R_{0}=R(\varphi, \psi)$, calculate the value of the goal function $\Phi_{0}=\Phi\left(R_{0}\right)$, create start temperature $T_{0}$ and lower limit of it $\varepsilon>0$. Repeat steps 2 5 while $T_{0}>\varepsilon$.

Step 2. Generate the new value of $R_{1}=R_{0}+\Delta R$, calculate the value of the goal function $\Phi_{1}=\Phi\left(R_{1}\right)$, calculate delta of the goal function $\Delta \Phi=\Phi_{1}-\Phi_{0}$.

Step 3. Test the condition $\Delta \Phi<0$. If it is true then take $R_{0}=R_{1}$, downgrade the temperature and go to Step 2, else Step 4.

Step 4. Generate the random number $k \in[0 ; 1]$, calculate the probability $P$ of accepting a new value,

$$
P\left(R_{0}=R_{1}\right)=\exp \left(-\Delta \Phi / T_{0}\right)
$$

Step 5. Test the condition $P>k$. If it is true then take $R_{0}=R_{1}$, downgrade the temperature and go to Step 2, else simple go to Step 2 .

Afterwards let us start second methods, whose general iterative scheme has the form

$$
R^{(q+1)}=R^{(q)}+e^{(q)} \cdot I^{(q)},
$$

where $I^{(q)}=-\Phi_{R}^{\prime}+j^{(q)} \cdot I^{(q-1)}, q$ is the iteration number; $e$ is the minimization step which is determined by onedimensional minimization of function $\Phi\left(R^{(q+1)}\right)$ with gold section method; $I^{(0)}=\Phi_{R}^{\prime} j^{(q)}$ is the coefficient of the CG method [17-20]; $\Phi_{R}^{\prime}$ is the Frechet derivative of functional (8), which is calculated as $[7,8]$

$$
\Phi_{R}^{\prime}=d \mu+\alpha d \Omega
$$

where $d \mu=2 \sum_{x_{M}} \sum_{y_{M}}\left\lfloor A(M, R)-U^{*}(M)\right\rfloor \cdot d A(M, R), d A$ is the derivative of the direct modeling operator (3); $d \Omega(R)=2\left(R(\varphi, \psi)-\frac{\partial^{2} R(\varphi, \psi)}{\partial \varphi^{2}}-\frac{\partial^{2} R(\varphi, \psi)}{\partial \psi^{2}}\right)$.

\section{THE NUMERICAL REALIZATION OF THE ALGORITHM}

Consider the features of the common formulas $(5,8)$. For the numerical realization of the integrals we will use the 7 th order Newton-Cotes quadrature. Thus let us create two grids with 49 elements for $\varphi$ and $\psi$ for it: $\varphi=0, h_{\varphi}, 2 h_{\varphi}, \ldots 48 h_{\varphi}, 2 \pi, \psi=0, h_{\psi}, 2 h_{\psi}, \ldots 48 h_{\psi}, \pi$. Thus we must to determinate 2401 unknown variables of $R(\varphi, \psi)$

For calculate first and second partials derivative let us use 5-th points difference scheme.

Let us consider the features of the first algorithm. We need to identify two patterns: the generation of a new point 
$\Delta R$ (here we have to consider the limitations $R \in(0 ; 1)$ ) and annealing temperature lowering formula. Let the value of $\Delta R$ is determined as [10] $R_{t+1}=R_{t}+0,1 \cdot$ rand and recalculate $R_{t+1}=$ rand, if $R_{t+1} \notin[0 ; 1]$, where the rand is uniformly distributed numbers in the interval $[0 ; 1]$.

Let the temperature will fall under by the Cauchy scheme as $T=T / \ln (1+t)$, where $t$ is the number of SA algorithm iteration.

Consider the features of the second algorithm. «The conjugate gradient method» is a common name for a variety of methods each realization of which is determined by the choice of the value $j^{(q)}$. In $[17-20]$ there are over 15 options of it. Experimentally, after several tests, the following formula was chosen as

$$
j^{(q)}=\max \left\{0 ; \min \left(\begin{array}{l}
\frac{\Phi_{q+1}^{\prime} \bullet \Phi_{q+1}^{\prime}}{\Phi_{q}^{\prime} \bullet \Phi_{q}^{\prime}} \\
\frac{\Phi_{q+1}^{\prime} \bullet\left(\Phi_{q+1}^{\prime}-\Phi_{q}^{\prime}\right)}{\Phi_{q}^{\prime} \bullet \Phi_{q}^{\prime}}
\end{array}\right)\right\}
$$

where $\bullet$ is the dot product, $q-$ is the number of CG algorithm iteration.

Let us apply this algorithm for test problem: failure zone is the ellipsoid with $A=100, B=75, C=25 \mathrm{~m} ; H=150$ $\mathrm{m}$; inclination angle $\gamma=30^{\circ}$ For simulation errors in real measurements let us add a random correction as $U=U^{e x}+0,5($ rand $-0,5)$. For the graphic image of the restored area let us use two sections of $R(\varphi, \psi): R\left(26 h_{\varphi}, \psi\right)$ and $R\left(\varphi, 26 h_{\psi}\right)$.

The result of calculation after $50 \mathrm{SA}$ iterations and $10 \mathrm{CG}$ iteration with double repeat of it is on Fig 4.

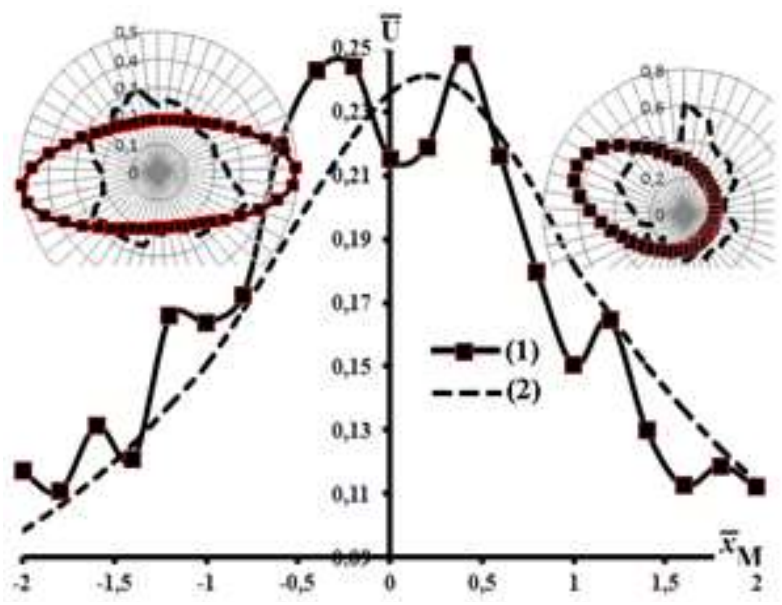

Fig. 4. The precise (1) and the results of restoration failure zone (2).

\section{CONCLUSION}

As in [1 - 3] let us give practical recommendations for using of the developed methodology. The user must do a coordinate grid on the surface with a step of about 25-50 meters. In each node of this grid, the user measure the field potential. The contour map is constructed, along which the elongation line of the proposed source is determined. More accurate measurements in the amount of at least 20 are taken along this line. These data will be used as initial data. If detailed measurements are impossible, then an approximate interpolation polynomial is constructed, along which the required values of the potential are determined. Further, with the help of the developed program, the form of the 3D failure zone is defined.

\section{REFERENCES}

[1] D. Sirota, V. Ivanov, V. Khyamyalyainen. Definition of the form of coal spontaneous combustion source as the inverse problem of geoelectrics [J]. E3S Web of Conferences, 2017, (15, 01012)

[2] D. Yu. Sirota, V.V. Ivanov. Diagnostics of the shape and orientation of a rock failure zone based on electrical measurements as an inverse problem of geophysics [J]. Russian Geology and Geophysics, 2018, (59), $105-109$

[3] D. Sirota, V. Ivanov. Determination of the geometric form of a plane of a tectonic gap as the inverse ill - posed problem of mathematical physics [J]. ]. E3S Web of Conferences, 2017, (21, 01017)

[4] V. V. Ivanov. Physical bases of electromagnetic processes at formation of the hearth of destruction in an array of rocks [M]. Kemerovo, KuzSTU, 1994.

[5] S. N. Zhurkov. Kinetic concept of strength of solids International Journal of Facture Mechanics [J], 1964, 1(4), 311-323.

[6] A.N. Tikhonov, V.B. Glasko. Approximate solution of Fredholm integral equation of the first kind $[\mathrm{J}]$. Journal of computational mathematics and mathematical physics, 1964, 4(3), $564-571$.

[7] A.N. Tikhonov, V.B. Glasko. Use of the regularization method in nonlinear problem $[\mathrm{J}]$. Journal of computational mathematics and mathematical physics, 1965, 5(3), $463-473$.

[8] V.A. Morozov. Regularization methods for ill-posed problems [M]. New York, CRC Press, 1993

[9] F.D.M. Neto, A.J.S. Neto. An introduction to inverse problems with application [M]. Dordrecht, Springer, 2013

[10] H.W. Engl, M. Hanke, A. Neubauer. Regularization of inverse problems [M]. London, Kluwer Academic Publishers, 1996

[11] C.R. Vogel. Computational methods for inverse problems [M].Montana, SIAM, 2002

[12] Y. Wang, A.G. Yagola, C. Yang. Optimization and regularization for computational inverse problems and applications [M]. Dordrecht, Springer, 2011

[13] R.C. Aster, B. Borchers, C.H. Thurber. Parameter estimation and inverse problems [M] Elsevier Academic Press, 2005

[14] G. Chavent, Nonlinear least squares for inverse problems. [M]. Dordrecht, Springer, 2009

[15] . A.B. Bakushinsky, M.Yu. Kokurin, A. Smirnova. Iterative methods for ill-posed problems [M]. Berlin, Walter de Gruyter GmbH \& Co., 2011

[16] K.M. Mullen. Continuous Global Optimization in R [J]. Journal of Statistical Software, 2014, 60 (6) 1 - 45

[17] Yang Xiang, Sylvain Gubian et al. Generalized Simulated Annealing for global optimization: The GenSA Package [J]. The R Journal, 2013, $5(1), 13-28$.

[18] W.W. Hager, H. Zhang. A new conjugate gradient method with guaranteed descent and efficient line search [J].SIAM Journal Optimisation, 2005, 16 (1), 170 - 192.

[19] W.W. Hager, H. Zhang. Algorithm 851: CG_DESCENT, a Conjugate Gradient Method with Guaranteed Descent [J], ACM Transactions on Mathematical Software, 2006, 32(1) 113 - 137.

[20] Y.H. Dai, Y. Yuan. An efficient hybrid conjugate gradient method for unconstrained optimization [J]. 2001, Annals of Operations Research, (103), $33-47$ a 